\title{
AplicaÇões MÓveis PARA O TURISMO Cultural: CAminhos de Santiago
}

\author{
Miguel Mazeda \\ Centro de Criatividade Digital, Escola das Artes, Universidade Católica Portuguesa, Portugal \\ Luís Teixeira \\ Centro de Investigação em Ciência e Tecnologia das Artes, Escola das Artes, Universidade Católica Portuguesa, Portugal
}

\begin{abstract}
RESUMO
Este artigo apresenta o resultado do estudo sobre as aplicações móveis na área do Turismo Cultural para os Caminhos de Santiago. Este estudo foi desenvolvido no âmbito da atividade B2 "Gestão de conteúdos pertencentes ao património cultural nacional baseado em sistemas abertos de preservação e interação" do projeto CHIC - "Cooperative Holistic View on Internet and Content" (POCI-O1-0247-FEDER-024498). A atividade B2 tem como objetivo conceber e implementar uma plataforma para o desenvolvimento de aplicações móveis, que suporte conteúdos de média avançados como realidade aumentada, vídeo $360^{\circ}$ e dados georreferenciados, tendo sido selecionada como prova de conceito o desenvolvimento de uma aplicação para os Caminhos de Santiago. Neste artigo são apresentadas e analisadas as principais caraterísticas das aplicações móveis existentes para os Caminhos de Santiago.
\end{abstract}

\section{Palavras-Chave}

aplicações móveis; Caminhos de Santiago; CHIC; realidade aumentada; turismo cultural

\section{MOBILE APPLICATIONS FOR CULTURAL TOURISM. ST. JAMES WAY}

\begin{abstract}
This article presents the result of the study on mobile applications (apps) in the area of Cultural Tourism for the St. James Way. This study was developed within the scope of activity B2 "Content management belonging to the national cultural heritage based on open systems of preservation and interaction" of the CHIC project - "Cooperative Holistic View on Internet and Content" (POCl-01-0247-FEDER-024498). Activity B2 aims to design and implement a platform for the development of mobile applications, which supports advanced media contents such as augmented reality, $360^{\circ}$ video and georeferenced data, with the development of an application for the St. James Way. In this article, the main characteristics of existing mobile applications for the St. James Way are presented and analyzed.
\end{abstract}

\section{KeYwORDS}

mobile apps; St. James Way; CHIC; augmented reality; cultural tourism 


\section{INTRODUÇÃo}

A Agência Nacional de Inovação (ANI), visa fomentar ações de apoio à inovação tecnológica e empresarial em Portugal, contribuindo para fortalecer o Sistema Nacional de Inovação (SNI) e para robustecer a competitividade da economia nacional nos mercados globais. Uma das ações que a ANI promove são os programas mobilizadores: projetos estratégicos de investigação e desenvolvimento que têm como objetivo a criação de novos produtos, processos ou serviços (PPS) com elevado conteúdo tecnológico e de inovação.

O projeto mobilizador CHIC - "Cooperative Holistic View on Internet and Content" (POCl-01-0247-FEDER-024498), visa a criação de um conjunto de serviços e plataformas necessários para proporcionar a produção e distribuição de novas formas de consumo de conteúdos, promovendo a qualidade dos serviços e da experiência de consumo. Está estruturado em vários PPS que integram plataformas abertas de gestão da produção e distribuição de conteúdos digitais na cloud e a gestão de conteúdos pertencentes ao património cultural nacional com base em sistemas abertos de preservação e interação, nomeadamente através da PTAPNC (Plataforma tecnológica de apoio ao Plano Nacional de Cinema), da NVDCTP (Novas Plataformas de Difusão de Conteúdos na área do Turismo e Património) e da ILN (Interfaces em linguagem natural para navegação online e em sistemas de distribuição por cabo).

$A$ atividade $B 2$ do projeto $\mathrm{CHIC}$ tem como objetivo desenvolver uma plataforma de difusão de conteúdos na área do Turismo e Património, integrando recursos jornalísticos que possibilitem aos utilizadores a descoberta do património cultural e histórico através de uma aplicação móvel. A aplicação vai suportar e fornecer diferentes tipos de conteúdos, desde artigos (de texto) contextualizados até experiências de realidade aumentada, realidade virtual e mixed reality'.

Este estudo foi realizado na fase inicial do projeto de forma a apoiar a definição de requisitos e funcionalidades da plataforma a desenvolver.

\section{APLICAÇões MÓVEIS: NOVAS FERRAMENTAS TURÍSTICAS}

O rápido crescimento do uso dos smartphones e das respetivas aplicações móveis criou novas formas da indústria do turismo se conectar aos seus visitantes enquanto estes viajam. Os smartphones foram rapidamente adotados como ferramentas de viagens turísticas (Dickinson et al., 2014). O desenvolvimento das tecnologias tornou-se inerente ao crescimento do turismo por ser um recurso que contribui para a promoção de destinos assim como para a obtenção de informação por parte dos turistas. Neste processo de inovação, as tecnologias tornam flexível todo o curso de uma viagem, com meios que permitem planear a mesma, assim como adequá-la às circunstâncias (Oh, Letho \& Park, 2009). Uma viagem é suportada por uma grande variedade de ferramentas tecnológicas através de diferentes plataformas, acessíveis em diferentes locais e datas (Lamsfus, Wang, Alzua-Sorzabal \& Xiang, 2015; Miller, 2012).

\footnotetext{
' Referida, por vezes, como realidade híbrida ou realidade mista. Mistura do mundo real e do virtual, de maneira a produzir
} novos ambientes e visualizações, em que os objetos físicos e digitais coexistem e interagem entre si. 
As aplicações fornecem resultados aos utilizadores mediante um conjunto de parâmetros variáveis definidos pelo próprio utilizador e pelo seu contexto. Por exemplo, os resultados de uma pesquisa no Google por restaurantes de um utilizador holandês serão diferentes no momento de planear a viagem quando está na Holanda ou quando visita outro país como Portugal. Apesar das consultas anteriores estarem armazenadas, - Google vai detetar a localização atual e atualizar as sugestões de palavras-chave. O crescimento exponencial de soluções de ambientes digitais torna as tarefas como a busca e seleção de informação pesadas. Sistemas de recomendação ${ }^{2}$ permitem reduzir esta sobrecarga de informação e oferecer recomendações baseadas nos perfis e hábitos dos utilizadores.

Os sistemas de recomendação disponíveis no turismo eletrónico adquirem as necessidades/desejos do utilizador de forma explícita ou implícita, pois estamos perante um ambiente propício a que a nossa atividade seja extraída (Gavalas, Konstantopoulos, Mastakas \& Pantziou, 2014). Desta forma, sugerem destinos a visitar, pontos de interesse através das buscas do utilizador, apresentando até descontos ou pacotes turísticos que vão de encontro ao histórico de pesquisa. O objetivo destes sistemas é facilitar a pesquisa do utilizador e simultaneamente persuadi-lo na aquisição dos serviços propostos.

O mercado das aplicações móveis está em constante expansão, como podemos constatar pelo consumo de smartphones e pelas notícias divulgadas pelos media. De acordo com Dias (2018), em 2018, em 2017, a nível mundial, o número de downloads foi cerca de 175 mil milhões e os gastos em aplicações móveis 70 mil milhões de euros. Estes valores representam crescimentos na ordem dos $60 \%$ e $105 \%$, respetivamente, em relação a 2015 (App Annie, 2018). No que toca ao turismo, assistimos a um crescimento significativo em Portugal devido a uma mudança no paradigma da oferta turística, sem que este fenómeno seja de origem conjuntural (Costa, 2017).

$\mathrm{Na}$ área do turismo existe uma participação ativa dos municípios no desenvolvimento de aplicações que promovam as cidades. É natural que num mundo em que uma grande parte da população usa smartphone (cinco mil milhões ${ }^{3}$ ) exista uma preferência por aplicações móveis que facilitem o acesso à informação e tornem a vida mais cómoda, prática e simples. Quando viajam este interesse mantêm-se, especialmente pelas aplicações georreferenciadas que fornecem informações sobre mapas, guias, serviços, pontos de interesse, eventos, entre outros.

Em Portugal, esta aposta é visível e vários municípios têm lançado aplicações móveis que têm servido para facilitar a mobilidade dos visitantes nas suas cidades. Guimarães, é um dos exemplos ao lançar a aplicação Guimarães Mobitur, em 2012, para receber a Capital Europeia da Cultura 2012.

Na mesma medida, e de forma crescente, os municípios têm apostado neste tipo de plataformas para melhorar a experiência do visitante, dispor de novas maneiras de atrair turistas, disponibilizar facilmente informações, reforçar a visibilidade da marca

\footnotetext{
${ }^{2}$ O TripAdvisor e o Zoomato são dois/duas websites/aplicações móveis que funcionam como sistemas de recomendação populares na área do turismo.

${ }_{3}^{3}$ Retirado de http://www.gsma.com/newsroom/press-release/number-mobile-subscribers-worldwide-hits-5-billion/
} 
local, facilitar o acesso a produtos e serviços de entidades locais de forma a potenciar a geração de maiores receitas assim como ter uma plataforma que permita receber feedback direto e mais rápido dos visitantes. No mundo hiperconcorrencial em que vivemos, é importante não ficar atrás da concorrência, oferecendo produtos atrativos, mas também itens funcionais e apelos emocionais.

\section{O uso de APlicaÇões móveis em PeRegrinaÇão até Santiago de Compostela}

Desde algumas décadas atrás, o turismo começou a fazer parte da vida de qualquer indivíduo, seja em que âmbito for com variados efeitos positivos (Glaesser, 2006). Desta forma, a indústria do turismo tem-se posicionado em lugares de liderança classificando-se como uma atividade económica relevante a nível global. De acordo com os requisitos da Unesco sobre o património cultural, Portugal foi estabelecendo um programa de incremento de projetos turísticos, com o objetivo de valorizar não só os monumentos, mas também as comunidades onde estão inseridos (Lopes, 2000). $\mathrm{O}$ turismo tem um crescimento praticamente ininterrupto ao longo do tempo, representando atualmente $7 \%$ das exportações mundiais de bens e serviços (World Tourism Organization [UNWTO], 2017).

O turismo cultural e religioso apresenta-se num lugar significativo da economia deste setor, principalmente em cidades e locais com uma presença relevante de património civile religioso, assim como manifestações no domínio da crença e respetivas práticas religiosas. A cultura tem como principal motivação o conhecimento e a existência de uma valorização. O seu conceito é basilar em várias áreas académicas, mas sempre houve dificuldade em defini-lo. Na sua origem etimológica, encontramos duas diferentes derivações. Colere significa cultivar no sentido de cultivar um campo, o que implica mudança e transformação, processos de fertilização do solo e crescimento (Pires, 2006). Cultus significa adorar, prestar culto no sentido de desenvolver um certo sentimento religioso, o que implica igualmente uma mudança - interior (Pires, 2006). Desta forma, percebemos uma ligação inerente entre a cultura e as práticas religiosas.

Ao longo dos anos, o conceito de cultura tem evoluído e têm sido várias as definições atribuídas a este conceito. De um modo geral, a cultura refere-se a um conjunto de processos e padrões aprendidos do comportamento humano, tais como a língua, a religião, os costumes e as convenções. De certa maneira, a cultura é o reflexo do desenvolvimento espiritual, intelectual e estético de uma sociedade, assim como do seu modo de vida (Pires, 2006). Pode-se afirmar que é um indicativo de progresso.

O património cultural e religioso une crenças a valores culturais, cruzando fortes aspetos arquitetónicos e visuais a razões espirituais. Desta maneira, o turismo cultural e religioso tem-se tornado relevante e apresentado fortes motivações para viajar (Antunes, 2016). Tendo o património cultural e religioso se colocado numa posição de destaque, o Ministério da Economia e do Emprego integra o património histórico, cultural, religioso e paisagístico no plano estratégico nacional do turismo apresentado em 2012. Um dos valores essenciais da marca "Destino Portugal" assenta na história, cultura e tradição. 
Existe uma necessidade em reforçar circuitos turísticos que destaquem a diversidade do património cultural, religioso e natural (Plano Estratégico Nacional de Turismo [PENT], 2012).

O turismo religioso e a constante aposta no mesmo é visível em todo o mundo. As regiões mais ricas em recursos históricos, religiosos e culturais têm utilizado isso em favor dos seus planos estratégicos. Por exemplo, o turismo religioso é a segunda maior indústria da Arábia Saudita, desde o início do século XXI e desde sempre que tem gerado receitas anuais de oito mil milhões e crescido a um ritmo mais rápido do que qualquer outro setor (Paul \& Mourad, 2017). Os governos ao tomarem medidas de inclusão perante locais e monumentos na lista de património mundial recebem benefícios diretos (financeiros e técnicos) por parte da Unesco, mas também alcançam prestígio e projeção internacional (Lopes, 2000). É de fácil compreensão que o aumento exponencial desta prática expõe os museus e monumentos a um grande desgaste assim como à própria banalização. Contudo, apesar desta sobrecarga negativa, mas normal dado o fluxo de turismo e o crescente interesse, que exige acessos restritos e sensibilização em relação ao respeito e à compreensão, existem benefícios como o favorecimento do respeito entre culturas, a criação de comunidades assim como a valorização económica.

Dado o crescimento turístico, também é crescente a preocupação das cidades e dos países. Esta preocupação prende-se, principalmente, em facilitar a vida de quem os visita, contribuindo para uma melhor experiência e complementando-a com uma presença digital dada a era em que vivemos.

No âmbito da peregrinação, mais propriamente no Caminho de Santiago, têm sido desenvolvidas várias aplicações móveis com o objetivo de facilitar a viagem dos peregrinos assim como o de completar a jornada de peregrinação através do desenvolvimento de conteúdos digitais.

Durante a peregrinação a Santiago de Compostela, os peregrinos podem usufruir da paisagem natural e cultural do Caminho. Contudo, e para além da contemplação, estamos perante o crescimento e a introdução dos meios digitais nestas práticas de origem medieval.

Vivemos numa era em que cada vez mais se assiste a uma ajuda da tecnologia móvel para planear uma viagem ou para tirar o máximo proveito no decorrer da mesma. Ao olhar para uma sociedade altamente tecnológica em que os peregrinos estão integrados, é importante perceber o uso tecnológico durante a peregrinação assim como a intenção de uso durante a mesma (Antunes, 2016).

De que forma difere a relação entre um peregrino e o uso de uma aplicação móvel durante a sua jornada da relação de um turista e o uso de uma aplicação móvel durante uma viagem? As motivações de um peregrino e de um turista são distintas. Para desenvolver uma aplicação móvel que tenha valor para peregrinos é crucial perceber o contexto de uma peregrinação e entender as especificidades do caminho de peregrinação. Apesar das diferenças antes de iniciarem uma viagem, existem várias caraterísticas em aplicações móveis para turistas que podem ser uteis para os peregrinos, como informações sobre o local onde se encontram, monumentos e locais de interesse na 
vizinhança, assim como locais para pernoitar e outras informações que confiram segurança e conforto.

Nos dias de hoje, normalmente, as pessoas têm mais do que uma razão para viajar (Drule, Chiş, Băcilă \& Ciornea, 2012). A literatura define duas categorias como forma de enquadrar as motivações para o turismo. Os push factors são estímulos internos que induzem as pessoas a viajar e preencher desta forma as suas necessidades enquanto que os pull factors são fatores externos especialmente relacionados com os atributos e a atratividade do destino (Crompton \& McKay, 1997; Drule, Chiş, Băcilă \& Ciornea, 2012; Kim, ChoongKi \& Klenosky, 2003; Morgan, 2006; Yoon \& Uysal, 2005). Estas categorias estão relacionadas e evoluem adaptando-se à situação. Da mesma forma, a motivação turística é um conceito complexo que depende de cada indivíduo e tem uma influência significativa no processo de decisão (Correia \& Crouch, 2004).

Num estudo recente, realizado com 222 peregrinos portugueses, verificou-se que um dos aspetos mais importantes de uma aplicação móvel sobre o Caminho de Santiago é a sua utilidade (Antunes \& Amaro, 2016). É crucial que esta apresente informações relevantes sobre o caminho tendo por base a localização do peregrino através de uma interface fácil de utilizar. Constatou-se um grande interesse de partilha de informação e experiências entre os peregrinos durante a jornada. No desenvolvimento de uma aplicação móvel para este mercado interessa desta forma implementar um mecanismo que permita essa partilha de conteúdos.

O uso da tecnologia durante a peregrinação é totalmente voluntário. Alguns peregrinos preferem libertar-se da tecnologia e aproximar-se da natureza assim como dar atenção às causas espirituais que os movem nesta jornada (Nickerson, Austreich \& Eng, 2014). Contudo, a existência de uma aplicação móvel com informações úteis sobre o Caminho de Santiago promove a intenção de uso da mesma (Antunes, 2016).

No tempo medieval o guia utilizado para realizar o Caminho de Santiago era o Codex Calixtinus, escrito em latim entre os anos 1130 e 1160 . Este, foi considerado o primeiro guia do Caminho. Hoje em dia, os peregrinos fazem-se acompanhar de vários guias de apoio existentes nas variadas línguas (Antunes, 2016). Está também ao dispor dos peregrinos a página oficial de apoio ao Peregrino (Oficina do Peregrino) que disponibiliza várias informações, nomeadamente os horários das atividades religiosas.

A utilização crescente de soluções tecnológica nos Caminhos de Santiago deve-se a este se ter tornado num percurso turístico, e pelo uso do GPS e partilha dos itinerários e fotografias por parte dos peregrinos.

\section{LEVANTAMENTO DE APLICAÇÕES MÓVEIS DISPONÍVEIS EM LOJAS ONLINE}

As aplicações existentes para o Caminho de Santiago são várias, verificando-se redundância nas funcionalidades que oferecem. Existe uma oferta vasta, principalmente para o sistema operativo (SO) Android. Algumas aplicações móveis suportam ambos os sistemas operativos.

Foram identificadas mais de 50 aplicações sobre o Caminho de Santiago. Para cada aplicação foi recolhida informação como: nome, programador, sistema operativo, tipo 
de aplicação e disponibilidade 4 . A seleção destes atributos foi feita com base em estudos, artigos, análise de questionários feitos a peregrinos e pesquisas nas lojas sobre o tema. Desta lista, procurou-se analisar quais as aplicações mais relevantes disponíveis nas lojas (App Store e Play Store) e as suas funcionalidades.

Desta forma, construíram-se duas tabelas (ver Apêndices) com informação como disponibilidade das aplicações nas lojas, tipo de aplicação predominante (online, offline, sincronização), onde se compreende a existência de 21 aplicações para iOS (uma indisponível) e 50 aplicações para Android (23 indisponíveis).

De um modo geral, as aplicações móveis sobre a peregrinação a Santiago de Compostela podem ser agrupadas em várias categorias pelo tipo de serviço que oferecem:

- informação geral;

- noticias e informação atual;

- acomodação;

- $\quad$ património cultural e locais turísticos;

- jogos, diário de viagem e livros;

- comunicação;

- tempo e previsão;

- $\quad$ saúde;

- $\quad$ interatividade (realidade aumentada).

A maioria das aplicações no mercado são completamente gratuitas, com a exceção de algumas que disponibilizam produtos pagos. No entanto, os preços não são elevados, e os artigos disponíveis nessas aplicações podem custar entre 0,79 €-5,90€. A única app que foge a esta regra é a "Camino Pilgrim - Frances" que permite donativos entre os 2,50 €-100 €, mas cuja instalação e usufruto são gratuitos. Estas aplicações não apresentam nenhuma secção de publicidade visível.

De acordo com o levantamento feito, a primeira análise foi o tipo de aplicação usada neste âmbito. O tipo online é predominante e deve-se à georreferenciação, ou seja, a app tem a informação em tempo real acerca da posição geográfica do portador do dispositivo móvel, o que permite às aplicações darem informações atualizadas e pertinentes considerando as coordenadas do mesmo.

Vivemos numa era tecnológica em que as tecnologias ao nosso dispor apresentam soluções interessantes para apresentar informação de variadas formas. Apesar destas facilidades, ainda não existem muitas aplicações sobre o Caminho de Santiago capazes de fazer o peregrino desfrutar da jornada no mundo digital e este mundo poder fazer parte da sua caminhada. As várias aplicações móveis sobre o Caminho de Santiago no mercado centram-se na funcionalidade e utilidade das informações que fornecem aos seus utilizadores. Estas pretendem dar a conhecer aos peregrinos aspetos que consideram importantes durante a jornada, como:

\footnotetext{
${ }^{4}$ Algumas aplicações listadas foram retiradas de estudos realizados anteriormente e, dessa forma, já não estão disponíveis. Também se referem como indisponíveis aquelas a que não se pode aceder nas lojas em Portugal.
} 
- informação específica para peregrinos que percorram o Caminho de Santiago a pé ou em bicicleta;

- posição do peregrino no mapa;

- planificador de etapas para organizar a rota de forma individualizada;

- pontos de informação geolocalizados sobre diferentes tipos de alojamentos, pontos de interesse e avisos importantes;

- informação meteorológica em tempo real e informação sobre localidades e serviços disponíveis;

- partilha da localização exata com amigos;

- acesso a diferentes itinerários.

Após a identificação das aplicações disponíveis nas lojas online (tabelas em apêndice) para o Caminho de Santiago decidimos focar o estudo num grupo menor de aplicações, mas que fosse representativo da oferta existente. Foram usados os seguintes critérios:

- avaliações pelos utilizadores/número de comentários;

- inquéritos realizados em estudos;

- conhecimento dos peregrinos sobre as aplicações existentes;

- seleção de uma app quando o mesmo programador disponibiliza várias aplicações para caminhos diferentes;

- uso de apenas uma das aplicações existentes nas lojas (App Store e Play Store).

\begin{tabular}{|c|c|c|c|c|}
\hline Nome & Programador & $\begin{array}{c}\text { SISTEMA } \\
\text { OPERATIVO } \\
\end{array}$ & Classificação & $\begin{array}{l}\text { NÚMERO DE } \\
\text { AVALIAÇÕES }\end{array}$ \\
\hline Guide of the Way of St James & Buen Camino & iOS & 4,3 & 10 \\
\hline Camino (Eroski Consumer) & BIKO & iOS & $N / D$ & $N / D$ \\
\hline A Wise Pilgrim Guide (Várias) & Wise Pilgrim & iOS & $N / D$ & $\mathrm{~N} / \mathrm{D}$ \\
\hline Augmented Reality St James Way & Ricardo Meana & Android & 3,7 & 33 \\
\hline Buen Camino & Chaligne Aurore & Android & 3,8 & 26 \\
\hline Caminho de Santiago Pro & Editorial Buen Camino & Android & 4,3 & 1204 \\
\hline Caminho de Santiago $360^{\circ}$ & IRALTA FILMS, S.L. & Android & 4,4 & 60 \\
\hline Cno. Santiago & CNIC & Android & 4,2 & 43 \\
\hline Camino de Santiago & COTESA & Android & 4,2 & 5 \\
\hline Camino de Santiago Guide & CaminoGuide.net & Android & 4,9 & 17 \\
\hline Camino de Santiago Guide v2.o & Tournride.com & Android & 3,3 & 131 \\
\hline Camino Francés - Wise Pilgrim & Wise Pilgrim & Android & 3,1 & 31 \\
\hline Camino Pilgrim - Francés & Aurea Moemke & Android & 4,8 & 1392 \\
\hline Caminos de Santiago (Várias) & IndependenTrip & Android & $\mathrm{N} / \mathrm{D}$ & $N / D$ \\
\hline Caminos de Santiago Eroski & Eroski Consumer & Android & 3,7 & 84 \\
\hline eCamino & eCamino Kft & Android & 3,3 & 26 \\
\hline miCamino & $\begin{array}{l}\text { micaminodesan- } \\
\text { tiago.com }\end{array}$ & Android & 4,3 & 1291 \\
\hline The Way of Saint James & SEGITTUR & Android & 4,1 & 19 \\
\hline
\end{tabular}


Usando os critérios definidos foram selecionadas 18 aplicações (Tabela 1)5. Algumas aplicações disponibilizam conteúdos média como realidade aumentada e fotografias $360^{\circ}$. A aplicação "Augmented reality St James Way" usa a realidade aumentada em pontos geolocalizados para disponibilizar informação sobre alojamentos e pontos de interesse. Neste caso, a informação é disponibilizada de forma tradicional não se tirando benefício do potencial de imersividade desta tecnologia. Ao analisar as funcionalidades das 18 aplicações observamos que as funcionalidades presentes em mais de $80 \%$ das aplicações são:

- informações sobre os pontos de interesse;

- diretório de alojamentos;

- diretório de localidades/serviços;

- $\quad$ rotas/itinerários a pé;

- distâncias/altitudes;

- GPS.

Observamos ainda que as funcionalidades presentes em mais de $10 \%$ e menos de 50\% das aplicações são:

- orientações/conselhos (conselhos para preparar o melhor possível a caminhada);

- informações sobre o caminho;

- meteorologia;

- multilingue;

- avaliação/comentários;

- rotas/itinerários bicicleta;

- planificador/programação personalizada;

- mapas offline (disponibilidade de descarga);

- partilha/envio redes sociais;

- partilha notas/fotografias;

- partilha/envio localização;

- lanterna.

As funcionalidades presentes em apenas uma aplicação de cada SO são:

- alertas em tempo real;

- diário (possibilidade de criação de um diário de peregrinação/inserção de elementos textuais e visuais em cada etapa de peregrinação);

- informações em realidade aumentada;

- perfil de utilizador (criação de um perfil com informação pessoal que permite interagir com outros peregrinos);

- guia interativo virtual (realização do caminho virtualmente através de guias interativos).

\footnotetext{
${ }^{5}$ Algumas aplicações para iOS não apresentam a classificação. A loja apenas disponibiliza dados a partir de um certo número de avaliações feitas. A classificação é feita na escala de o a 5 .
} 
Algumas das aplicações móveis selecionadas foram testadas relativamente à usabilidade. A usabilidade de uma aplicação móvel refere-se à facilidade de acesso ou esforço que leva um utilizador a atingir o seu objetivo. $O$ teste de usabilidade é um procedimento de verificação de funcionalidades da interface de uma plataforma digital, neste caso uma aplicação. Após a sua execução, é realizada uma análise de usabilidade e das principais dificuldades.

Os programadores devem ter atenção alguns aspetos na produção de uma aplicação, como o funcionamento da mesma em diferentes sistemas operativos e dispositivos. Em qualquer tipo de software, usabilidade é um conceito que considera três aspetos fundamentais: eficiência, aprendizagem e satisfação (Nayebi, Desharnais \& Abran, 2012). Neste sentido, ao avaliar a usabilidade de uma aplicação precisamos de considerar o tempo que demora a completar uma determinada tarefa, a aprendizagem das operações através da observação do objeto e o facto de ir de encontro às expectativas dos utilizadores.

A aplicação móvel "Caminho de Santiago Pro" foi desenvolvida a partir dos guias do Caminho de Santiago do reconhecido peregrino e jornalista Carlos Mencos (vencedor do prémio "Aymeric Picaud 2015" pela divulgação do Caminho de Santiago), que já conduziu dezenas de milhares de peregrinos a Santiago ${ }^{6}$. Os guias foram adaptados para serem incorporados na aplicação e tornaram-se mais completos que o próprio livro. A aplicação oferece, por predefinição, o guia do Caminho de Baztán. As outras rotas podem ser adquiridas por um preço inferior ao formato livro. Esta aplicação disponibiliza um elevado volume de dados, contudo pode ser confusa numa primeira abordagem pelas múltiplas janelas que têm de ser abertas pelo utilizador assim como pela disposição dos botões e conteúdos. O acesso à informação não é fácil sendo necessário o utilizador executar várias ações para conseguir ter acesso às informações. Quanto menor for o número de passos o utilizador tiver que executar, menor será o esforço do utilizador para atingir os seus objetivos e obter as informações que procura. A informação é disponibilizada em longas páginas o que dificulta o entendimento do conteúdo e atrasa a realização de ações na aplicação. A aplicação que tem como objetivo servir o peregrino e tornar a sua jornada facilitada torna-se num processo mais complexo que o esperado inicialmente. Contudo, apresenta vários fatores que contribuem para o seu sucesso como o grande número de opções de navegação assim como a inclusão dos guias do reconhecido Carlos Mencos, a credibilidade das informações e apoio imediato dado pelos programadores. A aplicação tem neste momento 1259 avaliações que totalizam uma classificação de $4,3 / 5^{7}$.

Outra aplicação selecionada para fazer o teste, foi a aplicação "Caminho de Santiago 360", a aplicação vencedora dos prémios "The App Tourism Awards 2016", organizados pela FITUR ${ }^{8}$ na categoria "Guia de Destino". Esta aplicação funciona como um

\footnotetext{
6 Informação retirada de http://periodistascompostela.gal/index.php/eventos/24-carlos-mencos-galardonado-premio-aymeric-picaud

7 Informação retirada de http://play.google.com/store?hl=pt_PT

${ }^{8}$ Feira Internacional de Turismo, realizada em Madrid, é o ponto de encontro global para profissionais de turismo e a
} 
guia interativo virtual que possibilita ao utilizador percorrer o caminho virtualmente, acedendo a informações sobre os pontos de interesse, assim como a possibilidade de visitar os monumentos através de fotografias e vídeos $360^{\circ}$ que colocam o utilizador no local. Nesta viagem interativa, o utilizador é acompanhado por Margaret, uma nova-iorquina apaixonada pela arte e pela arquitetura, e por António, um espanhol amigável que gosta de experimentar a culinária local, assim como por outros personagens. Esta aplicação desenvolvida pela IRALTA FILMS S. L. ${ }^{9}$ conta com 60 avaliações na Play Store e uma classificação final de $4,4 / 5^{10}$. Na versão gratuita apenas está disponível a primeira etapa: o itinerário de Roncesvalles a Pamplona. Os outros nove capítulos podem ser adquiridos individualmente por $1,00 €$ ou então pode-se adquirir o conjunto de todos os capítulos pelo valor de 2,09 €. A aplicação está bem estruturada com um design atual e acesso fácil à informação selecionada. A aplicação tem como objetivo explícito levar o caminho até ao utilizador. A disposição dos conteúdos permite uma navegação confortável e intuitiva. No primeiro capítulo, analisando a interação do utilizador com os dados disponíveis e a duração do vídeo $360^{\circ}$, o itinerário pode ser feito na aplicação num intervalo de tempo entre 10 a 15 minutos. Para os 10 capítulos existentes, o valor do intervalo deverá variar entre os 100 a 150 minutos de interatividade na aplicação.

Existem outras aplicações que não foram incluídas no estudo dada a sua reduzida expressão no mercado no momento deste estudo, mas que revelam potencial. Exemplo disso são duas aplicações lançadas pelos municípios e potenciam às estratégias dos mesmos. Os municípios representam um papel importante no desenvolvimento destes projetos, pois aumentam a presença digital das cidades e potenciam o crescimento do turismo.

A Junta da Galiza desenvolveu a aplicação móvel "Camiño de Santiago en Galicia", para Android e iOS, no âmbito do projeto "Smart Camiño". É uma aplicação orientada ao peregrino na sua viagem. Este projeto está incluído no "Plan Smart Turismo", no âmbito do "Plan Integral de Turismo", e tem um investimento esperado de 10 milhões de euros até 2020 (Xunta de Galicia, 2014). A Junta da Galiza, através da Agência de Turismo da Galiza desenvolveu o "Plano Integral de Turismo da Galiza" que pretende conferir sustentabilidade ao setor do turismo, adaptando os modelos de negócio e as políticas de gestão turística. O "Plano Smart Turismo" surge de maneira a impulsionar a modernização e a competitividade do setor através das TIC. Estes planos estão alinhados com o "Plano Estratégico da Galiza" e com a Estratégia Europa 2020, assim como os documentos preparatórios da "Estrategia de Especialización Inteligente Gallega" (RIS3)", um plano futuro para gerar bem-estar e emprego de forma sustentável e competir no exterior. Este investimento é também uma forma de atração turística e uma

\footnotetext{
principal feira de mercados recetivos e emissores da América Latina. Retirado de http://www.ifema.es/fitur_01/

9 Produtora audiovisual com foco nas experiências imersivas e uma paixão direcionada para a realidade virtual, está sediada em Madrid, Espanha. Retirado de http://iraltavr.es

${ }^{10}$ Retirado de http://play.google.com/store?hl=pt_PT

"Retirado de http://www.riszgalicia.es
} 
estratégia de oferta cultural, sabendo que o ano de 2021 é ano jacobeu ${ }^{12}$ ou ano santo compostelano. São anos que atraem muitos crentes pela procura da absolvição de todos os seus pecados.

A aplicação disponibiliza informações sobre todas as rotas do Caminho de Santiago desde a sua entrada na Galiza, assim como a rede oficial de albergues com todas as características, serviços e contactos, eventos, recursos turísticos, alertas de ofertas publicadas por estabelecimentos turísticos, condições meteorológicas fornecidas pela MeteoGalicia ${ }^{13}$ e dados de contacto e endereços. Este é um exemplo em que os custos da aplicação são suportados pelo município, e a aplicação faz parte de uma estratégia do mesmo.

No mesmo sentido, a "Valorização dos Caminhos de Santiago - Caminho Português da Costa" resulta de uma candidatura, conjunta dos 10 municípios incluídos na rota, ao Norte 2020 com o objetivo de valorizar e reconhecer oficialmente este Caminho como itinerário da peregrinação a Santiago'4.

A aplicação móvel "Caminhos de Santiago - Caminho Português da Costa" é desenvolvida pela Sétima Lda ${ }^{15}$, conta com a promoção dos municípios presentes no roteiro e com o financiamento do programa Norte 2020. A aplicação pretende funcionar como um guia interativo durante a caminhada do peregrino, permite descarregar o roteiro criado na plataforma web com todos os pontos de interesse a visitar e permite criar uma credencial digital. É uma aplicação útil também na fase anterior à caminhada, devido à lista de conselhos que incorporama aplicação com alusão ao vestuário, acessórios, calçado, mochila, pausas, técnicas para caminhar, alimentação e hidratação, cuidados com os pés, lesões e fadiga e alojamento.

A particularidade da credencial digital é curiosa, pois funciona como um perfil de utilizador que permite registar a pegada do peregrino submetendo conteúdos que mais tarde pode revisitar.

Esta aplicação permite a visualização e a partilha de testemunhos o que promove o diálogo sobre o Caminho Português da Costa, a partilha de experiências e dar a conhecer a novos peregrinos os pontos mais positivos assim como os negativos deste itinerário. Neste momento, a aplicação conta com 20 testemunhos o que demonstra a pouca atividade da mesma.

Apesar das poucas descargas efetuadas, os utilizadores apresentam níveis de satisfação elevados com a performance da mesma, facto visível nas 17 avaliações da loja Play Store que representam o 4,8/5 na classificação da aplicação.

\footnotetext{
${ }^{12}$ Estes anos acontecem quando o 25 de julho, festividade do martírio do apóstolo Santiago, coincide com um domingo. Com uma cadência de 6, 5, 6 e 11 anos, o 25 de julho ocorre num domingo.

${ }^{13}$ Agência meteorológica regional da Galiza, Espanha.

${ }^{14}$ Segundo alguns historiadores, este caminho era um dos eixos mais importantes para alcançar a casa do apóstolo em Santiago de Compostela. Este caminho a partir do Porto passando por Matosinhos, Maia, Vila do Conde, Póvoa de Varzim, Esposende, Viana do Castelo, Caminha, Vila Nova de Cerveira e Valença surge com a devida importância a partir do século XV. Retirado de http://www.caminhoportuguesdacosta.com/pt

${ }^{15}$ Empresa tecnológica que desenvolve soluções web a mobile, sediada em Braga. Retirado de http://www.setima.pt
} 
Esta aplicação está também disponível para o sistema iOS, mas o sistema de classificações da loja é mais rigoroso e só é apresentado a partir de um certo número de críticas. Contudo, é possível ver que foram feitas duas avaliações, ambas com classificação de $5 / 5$, com a referência à utilidade da aplicação antes e durante a peregrinação ${ }^{16}$.

É uma aplicação que apresenta elevado desempenho e uma rápida resposta na execução de tarefas. Está bem estruturada em termos de design e funcionalidade, assim como a nível de informação disponibilizada ao utilizador. Exibe apenas a informação essencial com uma navegação propícia à conclusão das tarefas, sem um número de passos exagerado nem um excessivo número de janelas abertas. A informação está disposta de forma clara e concisa o que reduz a necessidade de fazer zoom ou andar à deriva na plataforma.

Para além de aplicações móveis, existem outro tipo de plataformas digitais que já foram criadas no âmbito da peregrinação. A Autoridade Nacional de Proteção Civil (ANPC) criou uma plataforma digital para apoiar os peregrinos que se deslocaram a pé para Fátima. Foi lançada no âmbito da "Operação Fátima 2017" para a visita do papa a Fátima que ocorreu em maio desse mesmo ano ${ }^{17}$.

Esta plataforma reúne informação útil de apoio ao peregrino de Fátima e uma ferramenta de georreferenciação em que

o peregrino pode planear rotas e obter outras informações como pontos de apoio, locais de dormida, parques de estacionamento, áreas de refeições, postos de socorro entre outros recursos. ("Proteção Civil cria plataforma digital para apoiar os peregrinos", 2017)

Esta plataforma funcionou como um recurso de apoio à peregrinação, informando também sobre os cuidados a ter antes da peregrinação, durante a caminhada e na chegada ao destino. O Papa Francisco visitou Fátima nos dias 12 e 13 de maio de 2017 no âmbito das comemorações do centenário das aparições. Esta plataforma esteve ativa para este evento apenas. Os utilizadores tinham de efetuar um registo que permitia receber avisos importantes para uma peregrinação segura.

Num contexto universitário, no âmbito da promoção do turismo sustentável, iniciou-se, em 2018, um projeto universitário internacional em que Portugal está representado pela Universidade do Minho. O projeto "O valor dos direitos humanos no Caminho de Santiago: aproveitando o poder do turismo para promover o diálogo intercultural e a realização dos objetivos de desenvolvimento sustentável" explora o turismo como "instrumento de compreensão mútua e desenvolvimento sustentável" ("Pelo turismo sustentável nos Caminhos de Santiago", 2018). Neste projeto estão incluídas 20 universidades de 13 países (EUA, Peru, México, Sudão, Portugal, Espanha, Dinamarca, Luxemburgo, Holanda, Bélgica, Polónia, Montenegro e França). Numa fase inicial, os estudantes destas Universidades, trabalharam tendo como foco os principais princípios

\footnotetext{
${ }^{16}$ Informação retirada do website da Play Store e da App Store, em http://play.google.com/store?hl=pt_PT e http://www. apple.com/pt/ios/app-store/

${ }^{17}$ Retirado de http://www.protecaocivilfatima.pt
} 
e requisitos para o desenvolvimento do turismo sustentável bem como os princípios éticos e de responsabilidade associados ao Caminho de Santiago. Numa segunda fase, os estudantes têm a oportunidade de confrontar a análise teórica com a situação real da rota e perceber a viabilidade dos seus produtos, a sua sustentabilidade e capacidade de promoção do desenvolvimento local da rota ${ }^{18}$.

\section{DisCUSSÃo DE RESULTADOS E CONCLUSÃo}

Este projeto confronta-se com questões que procuram uma resposta que corresponda à era em que vivemos. A era digital é a era da prontidão e do momentâneo, a cada segundo aparece uma nova notificação, as atualizações de software são constantes e o ser humano quer estar sempre ligado (Palumbo, Dominici \& Basile, 2014). Estamos a criar dependências a um mundo virtual, a dependência de nos mostrarmos e a necessidade da aceitação social que revelam o ego enorme que vive dentro do ser humano.

Devido a esta exposição e a esta vontade de estar ligado, a oferta de apps tem vindo a crescer de forma significativa, aumentando o número de escolhas possíveis. Em 2000, já se constatava que os utilizadores da internet exibem uma impaciência notável e insistência na gratificação instantânea (Nielsen, 2000). A oferta de novas soluções digitais tem de ter em consideração as necessidades do utilizador e como promover o respetivo uso pelo público.

No universo das aplicações móveis, de acordo com a Fortune, num artigo escrito por Erin Griffith (2016), constatamos que em mais de $75 \%$ dos downloads de apps efetuados são executadas apenas uma única vez. Estes dados revelam que a maioria das aplicações não oferece uma boa experiência inicial ou não corresponde às expectativas dos utilizadores. Para ultrapassar estes problemas e as aplicações se tornarem num recurso essencial para o utilizador, estas precisam de assegurar requisitos de usabilidade relativamente ao design de aplicações móveis. É essencial perceber os objetivos do utilizador no contexto de utilização de forma a identificar as dificuldades que existem na execução das tarefas.

O objetivo do design é que este se torne invisível aos olhos do utilizador. É importante que a atenção do utilizador seja direcionada para a execução das tarefas. Estamos perante uma grande diversidade de tecnologias e dispositivos tecnológicos e, portanto, as aplicações devem-se adaptar aos requisitos funcionais dos dispositivos e dos sistemas operativos. Dessa forma e optando por normas de navegação criamos uma navegação mais intuitiva.

É importante compreender o contexto da utilização das aplicações pela indústria do turismo, o uso pelos turistas e educar a sua compreensão sobre as diferentes fases da sua viagem, assim como antecipar desenvolvimentos futuros (Lim, 2012). Desta forma será possível obter um melhor uso da aplicação móvel, por parte do utilizador e garantir melhores resultados. Podemos observar nas aplicações móveis analisadas no

\footnotetext{
${ }^{18}$ Retirado de http://affiliatemembers.unwto.org/content/activity-o
} 
teste de usabilidade, apresentaram algumas deficiências, como por exemplo a aplicação "Caminho de Santiago Pro", devido à profusão de ações necessárias para o utilizador conseguir aceder aos conteúdos.

Ângela Antunes (2016) entrevistou peregrinos e a maioria respondeu que leva dispositivos móveis com ligação à internet para a caminhada de peregrinação. Deste estudo demonstra que existe um interesse comum na existência de aplicações móvel sobre - Caminho de Santiago assim como o desejo por parte dos peregrinos do seu uso. $O$ conhecimento sobre as aplicações existentes é muito limitado e o seu uso ainda menor. Enquanto utilizadores de uma aplicação de apoio aos peregrinos de Santiago, as caraterísticas que os inquiridos valorizam mais estão diretamente ligadas ao Caminho, como:

- $\quad$ informações sobre as etapas;

- $\quad$ informações sobre os albergues;

- contactos urgentes;

- locais importantes;

- multilingue;

- offline;

- caraterísticas turísticas/culturais;

- conteúdos gerais sobre o Caminho.

Estas caraterísticas estavam presentes na maioria das aplicações disponíveis nas lojas online. Também são valorizados os conteúdos mais interativos e atrativos em termos de imagem, e são indicadas as caraterísticas religiosas (Antunes, 2016).

Os peregrinos manifestaram preferência por aplicações que funcionem em modo offline. No entanto no levantamento feito neste estudo constatou-se que a maioria das apps existentes funcionam apenas em modo online. Outro tópico é a reduzida informação em tempo real disponibilizada e a sua natureza (muito básica). Esta questão deverá ser aprofundada num estudo sobre modo de funcionamento (online, offline, sincronização) já que tem implicação em decisões como o armazenamento de dados, forma de acesso e disponibilização de informação, quando e como proceder a atualizações entre outras questões. A definição dos requisitos funcionais e operacionais deve também ter em conta os conteúdos a disponibilizar, e definir prioridades como por exemplo minimizar o tempo de acesso à informação, minimizar consumo de bateria, ou capacidade de armazenamento do dispositivo. Os sistemas móveis devem ser adaptados de uma forma eficaz que responda às necessidades do turista, fornecendo-lhe informações personalizadas (Oh, Lehto \& Park, 2009). Neste caso concreto, é imperativo pensar o contexto do peregrino e perceber como é possível fazê-lo continuar a viver a experiência de peregrinação num ambiente digital e numa situação em que o próprio pode criar as suas próprias experiências e partilhá-las com outros peregrinos.

No âmbito do turismo, verifica-se um interesse crescente pelo setor cultural e a adoção de aplicações móveis como ferramentas de apoio ao setor turístico. No contexto do projeto CHIC, na fase inicial do primeiro caso de demonstração do framework foram identificadas e analisadas aplicações móveis para os Caminhos de Santiago disponíveis 
nas lojas de aplicações. Existe uma grande oferta de aplicações sobre esta temática, mas nenhuma disruptiva no uso que faz de novos média como o uso de fotografia $360^{\circ}$, realidade aumentada ou conteúdos interativos. A única que se destaca pela inovação é a "Caminho de Santiago 360", mas a informação disponibilizada ao peregrino é limitada.

A maioria das aplicações funciona em modo online e apresenta características como como informações sobre as etapas/albergues, contactos urgentes, locais importantes, ser multilingue. Estas são as caraterísticas mais valorizadas pelos peregrinos com exceção do funcionamento online (preferência modo offline). O desenvolvimento de uma aplicação deverá garantir a existência destas funcionalidades identificadas. $O$ enfoque da aplicação a desenvolver deverá ser na produção de conteúdos digitais de elevada qualidade, componente muito limitada nas apps existentes atualmente.

\section{Agradecimentos}

Este artigo foi desenvolvido no âmbito do projeto NORTE-01-0145-FEDER-022133 e do projeto POCl-01-0247-FEDER-024498, cofinanciado pelo Programa Operacional Regional do Norte (NORTE 2O2O), através do Portugal2O20 e do Fundo Europeu de Desenvolvimento Regional (FEDER).

\section{REFERÊNCIAS}

Antunes, A. \& Amaro, S. (2016). Pilgrims' acceptance of a mobile app for the Camino de Santiago. In A. Inversini \& R. Schegg (Eds.), Information and communication technologies in tourism (pp. 509-521). Cham: Springer. https://doi.org/10.1007/978-3-319-28231-2_37

Antunes, Â. (2016). Caminhar na era tecnológica em direção a Santiago de Compostela: Estudo do uso de uma app por parte dos peregrinos. Dissertação de Mestrado, Escola Superior de Tecnologia e Gestão de Viseu, Portugal.

App Annie (2018). App Annie 2017 retrospective report. Retirado de https://www.appannie.com/en/insights/ market-data/app-annie-2017-retrospective/

Correia, A. \& Crouch, G. I. (2004). Tourist perceptions of and motivations for visiting the Algarve, Portugal. Tourism Analysis, 8(2), 165-169.

Costa, A. (2017, 17 de julho). Crescimento do turismo em Portugal deve-se a mudança no paradigma da oferta. Diário de Notícias. Retirado de https://www.dn.pt/lusa/crescimento-do-turismo-em-portugaldeve-se-a-mudanca-no-paradigma-da-oferta---antonio-costa-8643628.html

Crompton, J. L. \& McKay, S. L. (1997). Motives of visitors attending festival events. Annals of Tourism Research, 24(2), 425-439. https://doi.org/10.1016/So160-7383(97)80010-2.

Dias, M. P. (2018, 21 de janeiro). Gastamos cada vez mais dinheiro em aplicações móveis. Notícias ao Minuto. Retirado de https://www.noticiasaominuto.com/tech/940676/ gastamos-cada-vez-mais-dinheiro-em-aplicacoes-moveis

Dickinson, J. E., Ghali, K., Cherrett, T., Speed, C., Davies, N. \& Norgate, S. (2014). Tourism and the smartphone app: capabilities, emerging practice and scope in the travel domain. Current Issues in Tourism, 17(1), 84-101. 
Drule, A. M., Chiş, A., Băcilă, M. F. \& Ciornea, R. (2012). A new perspective of non-religious motivations of visitors to sacred sites: evidence from Romania. Procedia - Social and Behavioral Sciences, 62, 431-435. https://doi.org/10.1016/j.sbspro.2012.09.070

Gavalas, D., Konstantopoulos, C., Mastakas, K. \& Pantziou, G. (2014). Mobile recommender systems in tourism. Journal of Network and Computer Applications, 39, 319-333. https://doi.org/10.1016/j. jnca.2013.04.006

Glaesser, D. (2006). Tourism in a changing world. In D. Glaesser (Ed.), Crisis management in the tourism industry (pp. 1-8). Oxford: Elsevier.

Kim, S. S., ChoongKi, L. \& Klenosky, D. B. (2003). The influence of push and pull factors at korean national parks. Tourism Management, 24(2), 169-180.

Lamsfus, C., Wang, D., Alzua-Sorzabal, A. \& Xiang, Z. (2015). Going mobile: defining context for on-the-go travelers. Journal of Travel Research, 54(6), 691-701.

Lim, T. Y. (2012). Designing the next generation of mobile tourism application based on situation awareness. 2012 Southeast Asian Network of Ergonomics Societies Conference (SEANES) (pp. 1-7). Langkawi, Kedah, Malaysia. https://doi.org/10.1109/SEANES.2012.6299599

Lopes, F. (2000). O programa de incremento do turismo cultural - dos novos conceitos e motivações sobre o património cultural à criação de produtos turísticos de qualidade. Antropológicas, 4, 243-250.

Miller, C. C. (2012, 19 de setembro), Google updates android maps app as apple moves on. New York Times. Retirado de https://bits.blogs.nytimes.com/2012/o9/19/ google-updates-android-maps-app-as-apple-moves-on/

Morgan, M. (2006). Making space for experiences. Journal of Reital Q Leisure Property, 5(4), 305-313.

Nayebi, F., Desharnais, J.-M. \& Abran, A. (2012). The state of the art of mobile application usability evaluation. In 2012 25th IEEE Canadian Conference on Electrical and Computer Engineering (CCECE) (pp. 1-4). Montreal, QC, Canada. https://doi.org/10.1109/CCECE.2012.6334930

Nickerson, R., Austreich, M. \& Eng, J. (2014). Mobile technology and smartphone apps: a diffusion of innovations analysis. In Twentieth Americas Conference on Information Systems (pp. 1-12). Savannah.

Nielsen, J. (2000). Designing web usability: The pratice of simplicity. USA: New Riders Publishing.

Oh, S., Lehto, X. Y. \& Park, J. (2009). Travelers' intent to use mobile technologies as a function of effort and performance expectancy. Journal of Hospitality Marketing Q Management, 18(8), 765-781.

Palumbo, F., Dominici, G. \& Basile, G. (2014). The culture on the palm of your hand: how to design a user oriented mobile app for museums. In L. Aiello (Eds.), Handbook of research on management of cultural products: e-relationship marketing and accessibility perspectives (pp. 225-244). Hershey, PA, USA: IGI Global.

Paul, K. \& Mourad, M. (2017, O4 de setembro). Saudi arabi seeks islamic islamic tourism boost in test for heritage, tradition. Reuters. Retirado de https://www.reuters.com/article/ us-saudi-haj-tourism-idUSKCN1BF1XW

Pelo turismo sustentável nos Caminhos de Santiago (2018, 16 de março). Público. Retirado de https://www. publico.pt/2018/03/16/fugas/noticia/turismo-sustentavel-nos-caminhos-de-santiago-1806893

PENT, Plano Estratégico Nacional de Turismo. (2012). Plano estratégico nacional do turismo: horizonte 20132015. Retirado de http://www.turismodeportugal.pt / 
Pires, M. L. B. (2006). Teorias da cultura (2. ${ }^{a}$ ed.). Lisboa: Universidade Católica Editora.

Proteção Civil cria plataforma digital para apoiar os peregrinos (2017, 06 de abril). Jornal de Notícias. Retirado de https://www.jn.pt/nacional/protecao-civil-cria-plataforma-digital-para-apoiar-peregrinos-5775986. html

UNWTO, World Tourism Organization. (2017). UNWTO Tourism highlights: 2017 edition. Madrid: UNWTO. https://doi.org/10.18111/9789284419029

Uysal, M. \& Jurowski, C. (1994). Testing the push and pull factors. Annals of Tourism Research, 21 (4), 844-846. https://doi.org/10.1016/0160-7383(94)90091-4

Xunta de Galicia. (2014). Smart turismo: plan de desenvolvemento das TIC no eido do turismo. Retirado de https://transparencia.xunta.gal/c/document_library/get_file?folderld=687714\&name=DLFE-22454.pdf

Yoon, Y. \& Uysal, M. (2005). An examination of the effects of motivation and satisfaction on destination loyalty: a structural model. Tourism Management, 26(1), 45-56. https://doi.org/10.1016/j. tourman.2003.08.016

\section{APÊNDICES}

\begin{tabular}{|c|c|c|}
\hline Nome & Programador & Tipo \\
\hline Guide of the Way of St James & Buen Camino & Online \\
\hline Camino(Eroski Consumer) & $\mathrm{BIKO}$ & Online \\
\hline Camino Aragones - A Wise Pilgrim Guide & Wise Pilgrim & Online \\
\hline Camino de Invierno - A Wise Pilgrim Guide & Wise Pilgrim & Online \\
\hline Camino de Santiago - Bono Lacabus & Xunta de Galicia & Online \\
\hline Camino de Santiago - Camino Frances 2.0 & CNIG & Online \\
\hline Camino de Santiago del Sur a la Vía de la Plata. Sevilla - Mérida & Imagen MAS & Online \\
\hline Camino del Salvador - A Wise Pilgrim Guide & Wise Pilgrim & Online \\
\hline Camino Finisterre - A Wise Pilgrim Guide & Wise Pilgrim & Online \\
\hline Camino Frances - A Wise Pilgrim Guide & Wise Pilgrim & Online \\
\hline Camino Guide & Jack Fellows & Synch \\
\hline Camino Primitivo - A Wise Pilgrim Guide & Wise Pilgrim & Online \\
\hline CaminoDeSantiago & COTESA & Online \\
\hline eCamino & eCamino $\mathrm{Kft}$ & Synch \\
\hline Esoteric Camino France \& Spain & Sutro Media & Synch \\
\hline Le Puy / GR65 & AgenceTNT & Online \\
\hline TrekRight & Eugene Mallay & Online \\
\hline Via Plata IT & IndependenTrip & Online \\
\hline Visitabo Santiago de Compostela & ALHENA APP TRAVEL & Synch \\
\hline Way of St. James, St Jacobs Route Map & Dubbele.com & Synch \\
\hline
\end{tabular}




\begin{tabular}{|c|c|c|c|}
\hline NoME & PROGRAMADOR & TIPO & DISPONIBILIDADE \\
\hline Albergues_2.0 & HLE Aplicaciones & Offline & Indisponível \\
\hline Augmented Reality St James Way & Ricardo Meana & Online & Disponível \\
\hline Buen Camino & Chaligne Aurore & Offline & Disponível \\
\hline Caminho de Santiago Pro & Editorial Buen Camino & Online & Disponível \\
\hline Camino de Santiago $360^{\circ}$ & IRALTA FILMS S.L. & Online & Disponível \\
\hline Cno. Santiago & CNIG & Online & Disponível \\
\hline Camino de Santiago & COTESA & Online & Disponível \\
\hline Camino de Santiago Guide & CaminoGuide.net & Offline & Disponível \\
\hline Camino de Santiago Guide v2.o & Tournride.com & Online & Disponível \\
\hline Camino de Santiago HD & CNIG & Online & Indisponível \\
\hline Camino de Santiago my mobile & Miguel Angel Zamorano Porras & Online & Indisponível \\
\hline Camino de Santiago, Aragones & DeNAide & Online & Indisponível \\
\hline Camino de Santiago, Baztanes & DeNAide & Online & Indisponível \\
\hline Camino Frances - Wise Pilgrim & Wise Pilgrim & Online & Disponível \\
\hline Camino Frances IT & IndependenTrip & Online & Disponível \\
\hline Camino Pilgrim - Frances & Aurea Moemke & Online & Disponível \\
\hline Camino Portugues IT & IndependenTrip & Online & Disponível \\
\hline Camino Sanabres IT & IndependenTrip & Synch & Disponível \\
\hline Caminos de Santiago Eroski & Eroski Consumer & Online & Disponível \\
\hline Caminos de Santiago & IndependenTrip & Online & Disponível \\
\hline eCamino & eCamino Kft & Synch & Disponível \\
\hline ElCaminoenGPS_Burgos-Leon & Bluguîa, S.L. & Online & Indisponível \\
\hline EICAMINOenGPS_Galicia & Bluguîa, S.L. & Online & Indisponível \\
\hline ElCaminoenGPS_leon-Pedrafita & Bluguîa, S.L. & Online & Indisponível \\
\hline ElCaminoenGPS_Logroño-Burgos & Bluguîa, S.L. & Online & Indisponível \\
\hline ElCaminoenCPS_Pirineos-Logroño & Bluguîa, S.L. & Online & Indisponível \\
\hline Esoteric Camino France \& Spain & Sutro Media & Online & Indisponível \\
\hline Hotêis do Caminho de Santiago & Xose Zapata & Online & Disponível \\
\hline Le Puy / GR65 & AgenceTNT & Online & Disponível \\
\hline Los Caminos de Santiago & DeNAide & Online & Disponível \\
\hline my Camino de Santiago (mi Camino) & micaminodesantiago.com & Online & Disponível \\
\hline OCamiñoenGPS_Burgos-León & Bluguîa, S.L. & Online & Indisponível \\
\hline OCAMIÑOenGPS_Galicia & Bluguîa, S.L. & Online & Indisponível \\
\hline OCamiñoenGPS_León-Pedrafita & Bluguîa, S.L. & Online & Indisponível \\
\hline OCamiñoenGPS_Logroño-Burgos & Bluguîa, S.L. & Online & Indisponível \\
\hline OCamiñoenGPS_Pirineos-Logroño & Bluguîa, S.L. & Online & Indisponível \\
\hline Road to Santiago & Valerio Grosso & Online & Indisponível \\
\hline Santiago de Compostela & $\begin{array}{l}\text { Información e Comu- } \\
\text { nicación Local, SA }\end{array}$ & Online & Disponível \\
\hline Camino Santiago Bono lacobus & Xunta de Galicia & Online & Indisponível \\
\hline The Way of Saint James & SEGITTUR & Online & Disponível \\
\hline TheWayofSt)ames_PirineosLogroño & Bluguîa, S.L. & Online & Indisponível \\
\hline TheWayofSt)ames_Burgos-Leon & Bluguîa, S.L. & Online & Indisponível \\
\hline TheWayofSt)ames_León-Pedrafita & Bluguîa, S.L. & Online & Indisponível \\
\hline TheWayofSt)ames_Logroño-Burgos & Bluguîa, S.L. & Online & Indisponível \\
\hline TheWayofSt)amesinGPS_Galicia & Bluguîa, S.L. & Online & Indisponível \\
\hline Tu Camino & Pordefacto, s.l. & Online & Disponível \\
\hline
\end{tabular}




\begin{tabular}{llll}
\hline Ultreia! Camino Francés & Professor Lidenbrock & Online & Disponível \\
\hline Via de la Plata IT & IndependenTrip & Online & Disponível \\
\hline Visitabo Santiago Compostela & ALHENA APP TRAVEL & Synch & Disponível \\
\hline Camiño de Santiago en Galicia & Xunta de Galicia & Synch & Disponível \\
\hline
\end{tabular}

Tabela 2: Lista de aplicações móveis sobre os Caminhos de Santiago para Android

\section{NOTAS BIOGRÁFICAS}

Miguel Mazeda é colaborador do Centro de Criatividade Digital, tendo sido bolseiro de investigação no Projeto CHIC. Mestre em Gestão de Indústrias Criativas.

ORCID: https://orcid.org/0000-0002-1813-0650

Email:mmazeda@porto.ucp.pt

Morada: Universidade Católica Portuguesa, Escola das Artes, Centro de Criatividade Digital

Luís Teixeira é Professor da Escola das Artes da Universidade Católica Portuguesa. Coordenador Executivo do Mestrado em Gestão de Indústrias Criativas. Membro da direção da Escola das Artes. Doutorado em Engenharia Eletrotécnica e de Computadores.

ORCID: https://orcid.org/oooo-0002-1206-4576

Email: Iteixeira@porto.ucp.pt

Morada: Universidade Católica Portuguesa, Escola das Artes, Centro de Criatividade Digital

Submetido: 02/09/2019

Aceite: $20 / 11 / 2019$ 